arXiv version: fonts, pagination and layout may vary from GTM published version

\title{
Path concordances as detectors of codimension-one manifold factors
}

\author{
ROBERT J DAVERMAN \\ DENISE HALVERSON
}

\begin{abstract}
We present a new property, the Disjoint Path Concordances Property, of an ENR homology manifold $X$ which precisely characterizes when $X \times \mathbb{R}$ has the Disjoint Disks Property. As a consequence, $X \times \mathbb{R}$ is a manifold if and only if $X$ is resolvable and it possesses this Disjoint Path Concordances Property.
\end{abstract}

57N15, 57P05; 54B15, 57N70, 57N75

\section{Introduction}

Back in the 1950s R H Bing showed [1, 2] that his nonmanifold "dogbone space" is a Cartesian factor of Euclidean 4-space. Since then, topologists have sought to understand which spaces are factors of manifolds. It is now known (due to the work of Edwards [8] and Quinn [11]) that the manifold factors coincide with those ENR homology manifolds which admit a cell-like resolution by a manifold; equivalently, they are the ENR homology manifolds of trivial Quinn index. In particular, if $X$ has trivial Quinn index then $X \times \mathbb{R}^{k}$ is a manifold for $k \geq 2$. Whether $X \times \mathbb{R}$ itself is necessarily a manifold stands as a fundamental unsettled question.

Several properties of a manifold factor $X$ of dimension $n$ assure that its product with $\mathbb{R}$ is a manifold. Among them are:

(1) The singular (or nonmanifold) subset $S(X)$ of $X$ - namely, the complement of the maximal $n$-manifold contained in $X$ - has dimension at most $n-2$, where $n \geq 4$ (see the article by Cannon [3, Theorem 10.1]).

(2) There exists a cell-like map $f: M \rightarrow X$ defined on an $n$-manifold such that $\operatorname{dim}\left\{x \in X: f^{-1}(x) \neq\right.$ point $\} \leq n-3$ (see the article by Daverman [5, Theorem 3.3]).

(3) There exists a topologically embedded (n-1)-complex or an ENR homology (n-1)-manifold $K$ with $S(X) \subset K \subset X$ (see the book by Daverman [6, Corollaries 26.12A-12B]). 
(4) $X$ arises from a nested defining sequence, as defined by Cannon and Daverman [4] [6, Chapter 34], for the decomposition into point inverses induced by a cell-like map $f: M \rightarrow X$.

(5) $X$ has the Disjoint Arc-Disk Property of [6, page 193].

Condition (5) is implied by either (1) or (2) but not by (3) or (4).

More pertinent to issues addressed in this manuscript, Halverson [9, Theorem 3.4] proved that if an ENR homology $n$-manifold $X, n \geq 4$, has a certain Disjoint Homotopies Property, defined in the next section and abbreviated as DHP, then $X \times \mathbb{R}$ has the more familiar Disjoint Disks Property, henceforth abbreviated as DDP. Because the DDP characterizes resolvable ENR homology manifolds of dimension $n \geq 5$ as manifolds [8] [6, Theorem 24.3], it follows that $X \times \mathbb{R}$ is a genuine manifold if $X$ is resolvable and has this DHP. Still unknown are both whether all such ENR homology manifolds have DHP and whether $X$ having said DHP is a necessary condition for $X \times \mathbb{R}$ to be a manifold.

Since an ENR homology $n$-manifold $X, n \geq 4$, has DHP if it satisfies any of the properties mentioned in the second paragraph except (3), the sort of homology manifolds that might fail to have it are the ghastly examples of Daverman and Walsh [7]. Halverson [10] has constructed some related ghastly examples that do have DHP.

In hopes of better understanding the special codimension one manifold factors, this paper builds on Halverson's earlier work to present a necessary and sufficient condition, the Disjoint Path Concordances Property defined at the outset of Section 2, for $X \times \mathbb{R}$ to be a manifold (again provided $\operatorname{dim} X \geq 4$ ).

\section{Preliminaries}

Throughout what follows both $D$ and $I$ stand for the unit interval, [0,1]. A metric space $X$ is said to have the Disjoint Homotopies Property if any pair of path homotopies $f_{1}, f_{2}: D \times I \rightarrow X$ can be approximated, arbitrarily closely, by homotopies $g_{1}, g_{2}: D \times$ $I \rightarrow X$ such that

$$
g_{1}(D \times t) \cap g_{2}(D \times t)=\emptyset, \quad \text { for all } t \in I .
$$

Definition A path concordance in a space $X$ is a map $F: D \times I \rightarrow X \times I$ such that $F(D \times e) \subset X \times e, e \in\{0,1\}$.

Let $\operatorname{proj}_{X}: X \times I \rightarrow X$ denote projection. 
Definition A metric space $(X, \rho)$ satisfies the Disjoint Path Concordances Property $(D C P)$ if, for any two path homotopies $f_{1}, f_{2}: D \times I \rightarrow X$ and any $\epsilon>0$, there exist path concordances $F_{1}, F_{2}: D \times I \rightarrow X \times I$ such that

$$
F_{1}(D \times I) \cap F_{2}(D \times I)=\emptyset
$$

and $\rho\left(f_{i}, \operatorname{proj}_{X} F_{i}\right)<\epsilon$.

A homology n-manifold $X$ is a locally compact metric space such that

$$
H_{*}(X, X-\{x\} ; \mathbb{Z}) \cong H_{*}\left(\mathbb{R}^{n}, \mathbb{R}^{n}-\{\text { origin }\} ; \mathbb{Z}\right)
$$

for all $x \in X$. An ENR homology $n$-manifold $X$ is an homology $n$-manifold which is homeomorphic to a retract of an open subset of some Euclidean space (ENR is the abbreviation for "Euclidean neighborhood retract"); equivalently, $X$ is a homology $n$-manifold which is both finite-dimensional and locally contractible.

A homology $n$-manifold $X$ is resolvable provided there exists a surjective, cell-like mapping $f: M \rightarrow X$ defined on an $n$-manifold $M$. Quinn [11] has shown that (connected) ENR homology $n$-manifolds $X$, for $n \geq 4$, are resolvable if and only if a certain index $i(X) \in 1+8 \mathbb{Z}$ equals 1 .

A metric space $X$ has the Disjoint Arcs Property $(D A P)$ if every pair $f_{1}, f_{2}: D \rightarrow X$ of maps can be approximated, arbitrarily closely, by maps $g_{1}, g_{2}: D \rightarrow X$ for which $g_{1}(D) \cap g_{2}(D)=\emptyset$. All ENR homology $n$-manifolds, for $n \geq 3$, have the DAP.

The following Homotopy Extension Theorem is fairly standard. We state it here because it will be applied several times in our arguments.

Theorem 2.1 (Controlled Homotopy Extension Theorem (CHET)) Suppose $X$ is a metric ANR, $C$ is a compact subset of $X, j: C \rightarrow X$ is the inclusion map, and $\epsilon>0$. Then there exists $\delta>0$ such that for each map $f: Y \rightarrow C$ defined on a normal space $Y$, each closed subset $Z$ of $Y$, and each map $g_{Z}: Z \rightarrow X$ which is $\delta$-close to $\left.j f\right|_{Z}, g_{Z}$ extends to a map $g: Y \rightarrow X$ which is $\epsilon$-homotopic to $j f$. In particular, for any open set $U$ for which $Z \subset U \subset Y$, there is a homotopy $H: Y \times I \rightarrow X$ such that

(1) $H_{0}=j f$ and $H_{1}=g$

(2) $\left.g\right|_{Z}=g_{Z}$

(3) $\left.H_{t}\right|_{Y-U}=\left.j f\right|_{Y-U}$ for all $t \in I$

(4) $\operatorname{diam}(H(y \times I))<\epsilon$ for all $y \in Y$. 


\section{Main results}

In this section we will demonstrate that DCP characterizes codimension-one manifold factors among ENR homology $n$-manifolds, for $n \geq 4$, of trivial Quinn index. Essentially the DCP condition requires that any pair of level preserving path homotopies into $X \times I$ can be "approximated" by disjoint path concordances, where the approximation is measured in the $X$ factor. The following crucial proposition demonstrates that the DCP condition implies that any pair of level preserving path homotopies can be approximated, as measured in $X \times I$, by disjoint path concordances.

Proposition 3.1 Suppose that $(X, \rho)$ is a metric ANR with DAP. Then $X$ has DCP if and only if given any pair of level preserving maps $f_{1}, f_{2}: D \times I \rightarrow X \times I$ and $\epsilon>0$ there are maps $g_{1}, g_{2}: D \times I \rightarrow X \times I$ such that

(1) $f_{i}$ and $g_{i}$ are $\epsilon$-close in $X \times I$,

(2) $\left.g_{i}\right|_{D \times \partial I}$ is level preserving, and

(3) $g_{1}(D \times I) \cap g_{2}(D \times I)=\emptyset$.

Moreover, if $f_{1}(D \times \partial I) \cap f_{2}(D \times \partial I)=\emptyset$, then we also may require that $\left.g_{i}\right|_{D \times \partial I}=\left.f_{i}\right|_{D \times \partial I}$.

Proof Assume $X$ has DCP. Use $\widetilde{\rho}$ to denote the obvious sum metric on $X \times I$. Let $f_{1}, f_{2}: D \times I \rightarrow X \times I$ be level-preserving maps, and let $\epsilon>0$. Choose

$$
0=t_{0}<t_{1}<\cdots<t_{m}=1
$$

such that $t_{k}-t_{k-1}<\epsilon / 2$ for $k=1, \ldots, m$. Define

$$
J_{k}=\left[t_{k}, t_{k-1}\right], \quad f_{i}[k]=\left.f_{i}\right|_{D \times J_{k}} .
$$

Since $X$ has DAP then applying Theorem 2.1 (near $\operatorname{proj}_{X}\left(f_{1}(D \times I) \cup f_{2}(D \times I)\right)$ ) we may assume that $f_{1}\left(D \times t_{k}\right) \cap f_{2}\left(D \times t_{k}\right)=\emptyset$. Choose $\eta>0$ so that

$$
\widetilde{\rho}\left(f_{1}\left(D \times t_{k}\right), f_{2}\left(D \times t_{k}\right)\right)>\eta
$$

for $k=1, \ldots, n$. Let $\delta>0$ satisfy CHET for $X \times I$, for a small compact neighborhood $C$ of $f_{1}(D \times I) \cup f_{2}(D \times I)$ and $\min \{\eta / 2, \epsilon / 2\}$. Then by DCP, applied to the intervals $J_{k}$, there are maps $g_{i}[k]: D \times J_{k} \rightarrow X \times J_{k}$ satisfying

(1) $g_{i}[k]\left(D \times J_{k}\right) \subset C$,

(2) $\rho\left(\operatorname{proj}_{X} f_{i}[k], \operatorname{proj}_{X} g_{i}[k]\right)<\delta$,

(3) $\left.g_{i}[k]\right|_{D \times \partial J_{k}}$ is level preserving, and 
(4) $g_{1}[k]\left(D \times J_{k}\right) \cap g_{2}[k]\left(D \times J_{k}\right)=\emptyset$.

By CHET and the choice of $\delta$ there are maps $G_{i}[k]: D \times J_{k} \rightarrow X \times J_{k}$ satisfying

(1) $\rho\left(\operatorname{proj}_{X} f_{i}[k], \operatorname{proj}_{X} G_{i}[k]\right)<\epsilon / 2$,

(2) $\left.G_{i}[k]\right|_{D \times \partial J_{k}}=\left.f_{i}[k]\right|_{D \times \partial J_{k}}$, and

(3) $G_{1}[k]\left(D \times J_{k}\right) \cap G_{2}[k]\left(D \times J_{k}\right)=\emptyset$.

Set $G_{i}=\bigcup_{k} G_{i}[k]$. Confirming that $G_{1}$ and $G_{2}$ are the desired maps is straightforward.

The reverse direction is trivial. It merely requires treating any pair of path homotopies $D \times I \rightarrow X$ as level-preserving maps $D \times I \rightarrow X \times I$.

Definition A metric space $(X, \rho)$ satisfies the Disjoint 1-Complex Concordances Property ( $D C P^{*}$ ) if, for any two homotopies $f_{i}: K_{i} \times I \rightarrow X$, for $i=1,2$, where $K_{i}$ is a finite 1-complex, and any $\epsilon>0$ there exist concordances $F_{i}: K_{i} \times I \rightarrow X \times I$ such that

$$
F_{1}\left(K_{1} \times I\right) \cap F_{2}\left(K_{2} \times I\right)=\emptyset,
$$

$F_{i}\left(K_{i} \times e\right) \subset X \times e$ for $e \in \partial I$, and $\rho\left(f_{i}, \operatorname{proj}_{X} F_{i}\right)<\epsilon$.

Proposition 3.2 Suppose $X$ is a locally compact, metrizeable ANR with DAP. Then $X$ has DCP if and only if $X$ has DCP*.

Proof This argument is similar to the one showing the equivalence of the DDP with approximability of maps defined on finite 2-complexes by embedding [6, Theorem 24.1], and also to another one showing the equivalence of DHP (for paths) and a Disjoint Homotopies Property for 1-complexes [9, Theorem 2.9]. We supply the short proof for completeness.

To show the forward direction, endow $X$ with a complete metric $\rho$. Let $K_{1}$ and $K_{2}$ be finite 1-simplicial complexes, and define

$\mathcal{H}=\left\{\left(f_{1}, f_{2}\right) \in \operatorname{Map}\left(K_{1} \times I, X \times I\right) \times \operatorname{Map}\left(K_{2} \times I, X \times I\right):\left.f_{i}\right|_{D \times \partial I}\right.$ level-preserving $\}$

with the uniform metric. Note that $\mathcal{H}$ is a complete metric space and, therefore, a Baire space. For $\sigma_{i} \in K_{i}$, let

$$
\mathcal{O}\left(\sigma_{1}, \sigma_{2}\right)=\left\{\left(f_{1}, f_{2}\right) \in \mathcal{H}: f_{1}\left(\sigma_{1} \times I\right) \cap f_{2}\left(\sigma_{2} \times I\right)=\emptyset\right\} .
$$

Clearly $\mathcal{O}\left(\sigma_{1}, \sigma_{2}\right)$ is open in $\mathcal{H}$. To see that $\mathcal{O}\left(\sigma_{1}, \sigma_{2}\right)$ is dense in $\mathcal{H}$, let $\epsilon>0$. Choose $\delta>0$ to satisfy CHET for $X \times I$, for a small compact neighborhood of $\bigcup_{i} f_{i}\left(\sigma_{i} \times I\right)$, and $\epsilon$. Then choose $\eta>0$ to satisfy CHET for $X, \operatorname{proj}_{X}\left(\bigcup_{i} f_{i}\left(\sigma_{i} \times I\right)\right)$ 
and $\delta$. By Proposition 3.1 there are $\eta$-approximations $g_{i}$ to $\left.f_{i}\right|_{\sigma_{i} \times I}$ that are disjoint path concordances. First apply CHET to extend $g_{i}$ over $\left(\sigma_{i} \times I\right) \cup\left(K_{i} \times \partial I\right)$ so that the new $g_{i}$ is level preserving on $K_{i} \times \partial I$ and $\delta$-close to $\left.f_{i}\right|_{\left(\sigma_{i} \times I\right) \cup\left(K_{i} \times \partial I\right)}$. Then apply CHET again to extend $g_{i}$ over $K_{i} \times I$ so that $g_{i}$ is now $\epsilon$-close to $f_{i}$. Thus, $\mathcal{O}\left(\sigma_{1}, \sigma_{2}\right)$ is dense in $\mathcal{H}$. Since $\mathcal{H}$ is a Baire space,

$$
\mathcal{O}=\bigcap_{\left(\sigma_{1}, \sigma_{2}\right) \in K_{1} \times K_{2}} \mathcal{O}\left(\sigma_{1}, \sigma_{2}\right)
$$

is dense in $\mathcal{H}$. Note that if $\left(f_{1}, f_{2}\right) \in \mathcal{O}$ then $f_{1}\left(K_{1} \times I\right) \cap f_{2}\left(K_{2} \times I\right)=\emptyset$. Hence, $X$ has DCP*.

The other direction is trivial.

Definition A topography $\Upsilon$ on $D \times I$ consists of the following elements:

(1) A set $\left\{J_{1}, \ldots, J_{m}\right\}$ of consecutive intervals in $\mathbb{R}$ such that $J_{j}=\left[t_{j-1}, t_{j}\right]$ where $t_{0}<t_{1}<\cdots<t_{m}$.

(2) A finite set $\left\{L_{0}, \ldots, L_{m}\right\}$ of complexes embedded in $D \times I$ of dimension at most one. These complexes are called the transition levels.

(3) A finite set $\left\{K_{1}, \ldots, K_{m}\right\}$ of 1-complexes. These complexes are called the level factors.

(4) A set $\left\{\phi_{j}: K_{j} \times J_{j} \rightarrow D \times I\right\}_{j=1, \ldots, m}$ of maps which satisfy the following conditions:

(a) $L_{0}=\phi_{1}\left(K_{1} \times\left\{t_{0}\right\}\right)$

(b) $L_{m}=\phi_{m}\left(K_{m} \times\left\{t_{m}\right\}\right)$

(c) $L_{j}=\phi_{j}\left(K_{j} \times\left\{t_{j}\right\}\right) \cup \phi_{j+1}\left(K_{j+1} \times\left\{t_{j}\right\}\right)$ for $j=1, \ldots, m-1$

(d) $\left.\phi_{j}\right|_{K_{j} \times \text { int } J_{j}}$ is an embedding for each $j=1, \ldots, m$

(e) $\bigcup_{j=1}^{m} \operatorname{im}\left(\phi_{j}\right)=D \times I$

It is shown by Halverson [9] that a p.l. general position approximation of the projection of a map $f: D \times I \rightarrow X \times \mathbb{R}$ to the $\mathbb{R}$ factor induces a topographical structure on the domain $D \times I$ of $f$.

Definition A map $f: D \times I \rightarrow X \times \mathbb{R}$ is a topographical map if there is a topography, $\Upsilon$, on $D \times I$ which is level preserving in the sense that for each map $\phi_{j}: K_{j} \times J_{j} \rightarrow D \times I$ of the topography, $f \circ \phi_{j}\left(K_{j} \times t\right) \subset X \times t$ for all $t \in J_{j}$.

Note that a topographical structure on $D \times I$ is in no way related to the product structure of $D \times I$. The proof of the following lemma is provided in [9, Theorem 3.3]. 
Lemma 3.3 Every map $f: D \times I \rightarrow X \times \mathbb{R}$ can be approximated by a topographical map $g: D \times I \rightarrow X \times \mathbb{R}$.

Theorem 3.4 (Disjoint Concordances Theorem) Suppose $X$ is a locally compact, metric ANR with DAP. Then $X$ has DCP if and only if $X \times \mathbb{R}$ has DDP.

Proof $(\Leftarrow)$ Given two path homotopies $f_{1}, f_{2}: D \times I \rightarrow X$, treat them as levelpreserving maps $f_{1}, f_{2}: D \times I \rightarrow X \times I$. Applying DAP, and using CHET as before, we may assume without loss of generality that $f_{1}(D \times \partial I) \cap f_{2}(D \times \partial I)=\emptyset$. Since $X \times(0,1)$ has DDP, each $f_{i}$ can be approximated, fixing the actions on $D \times\{0,1\}$, by an $\epsilon$-close map $g_{i}$, such that the images of $g_{1}, g_{2}$ are disjoint. The DCP follows.

$(\Rightarrow)$ Given maps $f_{1}, f_{2}: I^{2}=D \times I \rightarrow X \times \mathbb{R}$, by Lemma 3.3 we may assume that each $f_{i}$ is a topographical map with topography $\Upsilon[i]$. An object $O$ in the definition of $\Upsilon[i]$ will be denoted as $O[i]$. Note that we also may assume the following:

(1) The set of intervals $\left\{J_{j}[i]\right\}$ are the same for $i=1,2$. This follows from subdividing the intervals appropriately as outlined in [9, Theorem 3.4].

(2) $f_{1}\left(\bigcup L_{j}[1]\right) \cap f_{2}\left(\bigcup L_{j}[2]\right)=\emptyset$. This follows from DAP and CHET.

By DCP* there exist maps $\psi_{j}[i]: K_{j}[i] \times J_{j}$, approximating $\phi_{j} f_{i}$, such that

$$
\operatorname{im} \psi_{j}[1] \cap \operatorname{im} \psi_{j}[2]=\emptyset .
$$

Applying Theorem 2.1, we may assume that

$$
\left.\psi_{j}[i]\right|_{K_{j}[i] \times \partial J_{j}}=\left.\phi_{j} f_{i}\right|_{K_{j}[i] \times \partial J_{j}} .
$$

(Recall that $\phi_{j} f_{i}\left(K_{j}[i] \times \partial J_{j}\right) \subset\left(L_{j} \cup L_{j-1}\right)$.) Set $g_{i}=\bigcup_{j} \psi_{j}[i]$. Then $g_{1}$ and $g_{2}$ are the desired disjoint approximations of $f_{1}$ and $f_{2}$.

Corollary 3.5 An ENR homology $n$-manifold $X$, for $n \geq 4$, has DCP if and only if $X \times \mathbb{R}$ has DDP.

When $n=3$, Corollary 3.5 is formally but vacuously true: since no ENR homology 4-manifold has DDP, no such homology 3-manifold can have DCP.

Corollary 3.6 Let $X$ be an ENR homology $n$-manifold, $n \geq 4$. Then $X \times \mathbb{R}$ is a manifold if and only if $X$ has trivial Quinn index and satisfies DCP.

Theorem 3.4 demonstrates the equivalence in $X \times \mathbb{R}$ of DCP and DDP. By the standard combination of the work of Edwards [8] and of Quinn [11], $X \times \mathbb{R}$ is a manifold if and only if it has both this latter property and trivial Quinn index. Furthermore, by [11], $i(X \times \mathbb{R})=i(X)$, without regard to any of these general position properties. 
Robert J Daverman and Denise Halverson

\section{Questions}

(1) Is every finite-dimensional Busemann space (see Thurston [12]) $X$ necessarily a manifold? Actually, there are two unsettled questions here: is $i(X)$, the Quinn index, trivial? When $\operatorname{dim} X>4$, must $X$ have DDP? It may be of interest to add that $X$ is known to be a manifold if $\operatorname{dim} X \leq 4$ [12].

The same pair of concerns crops up in the following setting.

(2) Suppose, for any two points $p, q$ of the compact, finite-dimensional metric space $X$, there is a homeomorphism from the suspension of a space $Y$ onto $X$ that carries the suspension points onto $p, q$. Is $X$ a manifold?

(3) Given an ENR homology $n$-manifold $X$, for $n \geq 4$, can maps $f, g: I^{2} \rightarrow X$ be approximated by $F, G: I^{2} \rightarrow X$ for which there exists a 0-dimensional $F_{\sigma}$ set $T \subset I^{2}$ such that

$$
F\left(I^{2}-T\right) \cap G\left(I^{2}-T\right)=\emptyset ?
$$

If so, $X \times \mathbb{R}$ will satisfy the DDP. Recall that $X \times \mathbb{R}$ satisfies the DDP when $S(X)$, the singular set of $X$, is at most $(n-2)$-dimensional. A key to the argument is that then one can obtain $F, G: I^{2} \rightarrow X$ and a compact, 0 -dimensional $T$ with $F\left(I^{2}-T\right) \cap G\left(I^{2}-T\right)=\emptyset$. Similar reasoning applies with $F_{\sigma}$-subsets $T$ in place of compact subsets. It may be worth noting that, because $X$ does satisfy the DAP, one can easily obtain (maps $F, G$ and) such a $T$ which is a $0-$ dimensional $G_{\delta}$-subset of $I^{2}$, but the argument requires a 0 -dimensional $F_{\sigma}$-subset, which is more "meager".

\section{References}

[1] $\mathbf{R} \mathbf{H}$ Bing, A decomposition of $E^{3}$ into points and tame arcs such that the decomposition space is topologically different from $E^{3}$, Ann. of Math. (2) 65 (1957) 484-500 MR0092961

[2] $\mathbf{R} \mathbf{H}$ Bing, The cartesian product of a certain nonmanifold and a line is $E^{4}$, Ann. of Math. (2) 70 (1959) 399-412 MR0107228

[3] J W Cannon, Shrinking cell-like decompositions of manifolds. Codimension three, Ann. of Math. (2) 110 (1979) 83-112 MR541330

[4] J W Cannon, R J Daverman, A totally wild flow, Indiana Univ. Math. J. 30 (1981) 371-387 MR611226

[5] R J Daverman, Detecting the disjoint disks property, Pacific J. Math. 93 (1981) 277-298 MR623564

Geometry \& Topology Monographs 9 (2006) 
[6] R J Daverman, Decompositions of manifolds, Pure and Applied Mathematics 124, Academic Press, Orlando, FL (1986) MR872468

[7] R J Daverman, J J Walsh, A ghastly generalized n-manifold, Illinois J. Math. 25 (1981) 555-576 MR630833

[8] R D Edwards, The topology of manifolds and cell-like maps, from: "Proceedings of the International Congress of Mathematicians (Helsinki, 1978)", Acad. Sci. Fennica, Helsinki (1980) 111-127 MR562601

[9] D M Halverson, Detecting codimension one manifold factors with the disjoint homotopies property, Topology Appl. 117 (2002) 231-258 MR1874088

[10] D M Halverson, 2-ghastly spaces with the disjoint homotopies property: the method of fractured maps, Topology Appl. 138 (2004) 277-286 MR2035486

[11] F Quinn, An obstruction to the resolution of homology manifolds, Michigan Math. J. 34 (1987) 285-291 MR894878

[12] P Thurston, 4-dimensional Busemann G-spaces are 4-manifolds, Differential Geom. Appl. 6 (1996) 245-270 MR1408310

Department of Mathematics, University of Tennessee, Knoxville

Tennessee 37996-1300, USA

Department of Mathematics, Brigham Young University

Provo, Utah, 84602, USA

daverman@math.utk.edu, deniseh@math.byu.edu

Received: 13 August 2003 